\title{
Closely Spaced Target Detection using Intensity Sorting-based Context Awareness
}

\author{
Sungho Kim ${ }^{\dagger}$ and Jin-Ju Won*
}

\begin{abstract}
Detecting remote targets is important to active protection system (APS) or infrared search and track (IRST) applications. In normal situation, the well-known constant false alarm rate (CFAR) detector works properly. However, decoys in APS or closely spaced targets in IRST degrade the detection capability by increasing background noise level in the CFAR detector. This paper presents a context aware CFAR detector by the intensity sorting and selection of background region to reduce the effect of neighboring targets that lead to incorrect estimation of background statistics. The existence of neighboring targets can be recognized by intensity sorting where neighboring targets usually show highest ranks. The proposed background statistics (mean, standard deviation) estimation method from median local pixels can be aware of the background context and reduce the effects of the neighboring targets, which increase the signal-to-clutter ratio. The experimental results on the synthetic APS sequence, real adjacent target sequence, and remote pedestrian sequence validated that the proposed method produced an enhanced detection rate with the same false alarm rate compared with the hysteresis-CFAR (H-CFAR) detection.
\end{abstract}

Keywords: Context aware, Target detection, Infrared, Intensity sorting, Constant false alarm rate

\section{Introduction}

In surveillance applications, remote targets are projected to an image plane. If the imaged target sizes are smaller than the instantaneous field of view (IFOV), the targets are called point targets. Optical dispersion and sensing mechanism make them Gaussian-like spread blobs, which are called small targets. If the target area is larger than approximately 100 pixels, the targets are called extended targets. Automatic target recognition methods cannot be applicable because there is no shape information except circular blob. Automatic target detection methods can localize the positions of remote small targets. Small target detection methods can be used in military surveillance problems such as active protection system (APS) and infrared search and track (IRST) to protect tanks and ships from attacking missiles $[1,2]$.

Previous researches focused on the issues of how to increase the detection rate of dim targets or how to reduce false alarms caused by environmental clutters in ground, sky, and sea. The issue of dim target detection was alleviated using the track-before-detect technique, which uses tracking to integrate target intensities in multi-frames before decision making for upgraded signal strength. The other issue of reducing false alarms can be solved by either spatial filters [3-5] or temporal filters [6-8]. Cloud

\footnotetext{
$\dagger$ Corresponding Author: Dept. of Electronic Engineering, Yeungnam University, Korea. (sunghokim@ynu.ac.kr)

* Department of Electronic Engineering, Yeungnam University, Korea (dnjswlswn1101@hanmail.net)

Received: September 9, 2015; Accepted: May 24, 2016
}

clutter around edge produces huge number of false detections, which can be reduced using the mean filter [9], median filter [9], least mean square filter [10, 11], and morphological Top-Hat filter [5, 12]. In sea-based IRST application, glints of sea surface generate flickering false alarms, which can be alleviated using the three dimensional fast Fourier transform spectrum [13], wavelet transform [14-16], low pass filter [17], and adaptive high pass filter $[18]$ in frequency domain analysis.

These approaches works well in their specific operational environments to reduce the number of false detections. The number of targets can be a single or multiple targets that were separated widely. In real scenarios such as APS and IRST, incoming remote multiple targets are located closely in image space due to the projection process. The previous methods frequently use a constant false alarm rate (CFAR) detector after specific filtering to maintain the number of false alarms in cluttered environments. The CFAR detector assumes that a single target exists in front of background clutters. If the condition fails, the CFAR cannot work properly. One of the examples is the scenario of closely spaced multi-targets, which occurs frequently in the APS or IRST applications as shown in Fig. 1. In APS, Fig. 1(a) sequence contains two small targets, a decoy on the left and a true missile on the right. In IRST, Fig. 1(b) sequence demonstrates the passing-by scenario of four neighboring group targets moving in the air. The original CFAR detector uses background statistics to set a detection threshold. In this paper, the closely spaced multi-target detection problem is solved by the proposed context aware CFAR (CA-CFAR) 

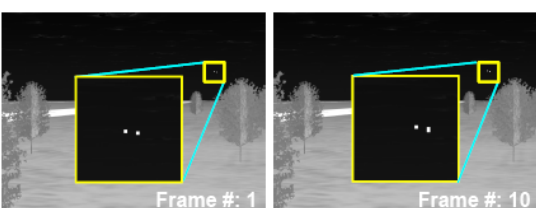

(a)
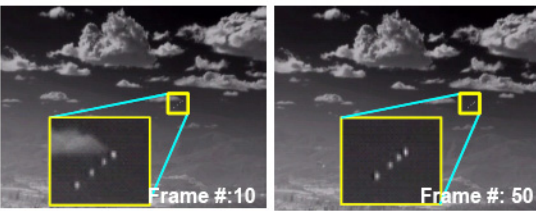

(b)
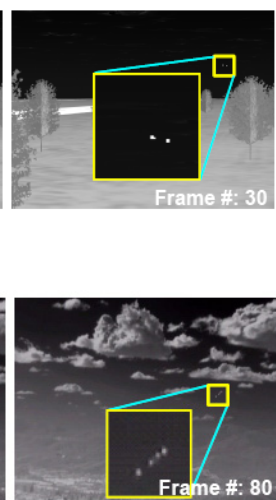

Fig. 1. Closely spaced multi-target detection scenarios in small infrared target detection: (a) incoming multitargets in active protection system scenario; (b) passing-by grouped targets in infrared search and track scenario.

detection by inserting an intensity ordering and pixel sampling block in front of the estimation of the background statistics, which can analyze the context of background status around a probing target. Until now, no one has proposed a suitable small target detection method for closely spaced multiple targets in the IRST or APS problem. Only Fernandez attempted to solve a similar problem using super-resolution processing [19].

Section 2 introduces the baseline detection method (HCFAR) and its limitation by a comparison with the wellknown CFAR detector. Section 3 presents the proposed closely spaced multi-target detection algorithm. The performance of this method is evaluated in Section 4 and the conclusions are reported in Section 5.

\section{Background of CFAR-based Small Infrared Target Detection}

CFAR-based detection method: The basic concept of CFAR-based detection is based on the automatic threshold selection using background statistics information. In cluttered environments, conventional infrared small target detection methods use the spatial filtering (signal enhancement and noise/clutter reduction) followed by a CFAR thresholding because of its robustness to clutter [20]. Fig. 2 summarizes the flow of conventional small target detection using CFAR method.

Given a test image (Fig. 2(a)), spatial filtering methods were applied to reduce the background clutter or enhance the targets (Fig. 2(b)). 3D perspective plots demonstrate the effect of background clutter reduction by the spatial filters. The original CFAR method regards each pixel as candidate target location and probes each pixel by sliding. Fig. 2(c) shows an example of probing region, which consists of a target region, a guard region, and a background region.

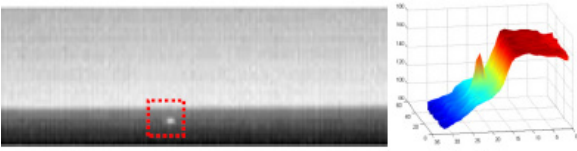

(a)

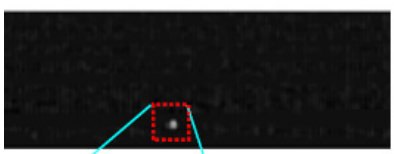

(b)
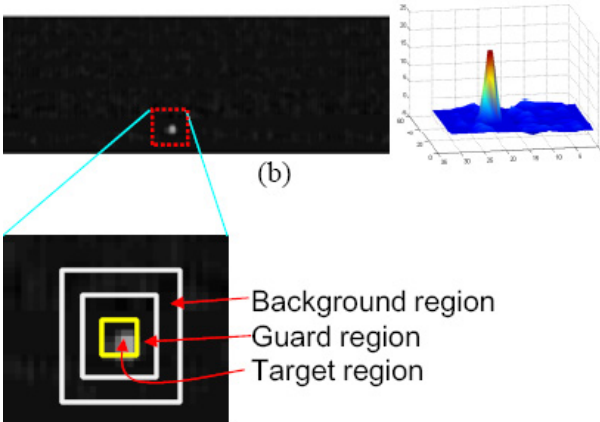

(c)

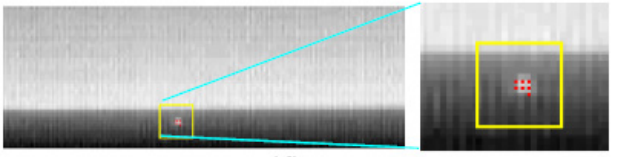

(d)

Fig. 2. CFAR-based small target detection flow: (a) test image; (b) result of spatial filtering; (c) example of target region and background region; (d) final detection using CFAR

Through the estimation of background statistics and adaptive thresholding (CFAR), the final targets are detected, as shown in Fig. 2(d) where red dots represent survived pixels.

Previous studies focused on the filtering methods in spatial or temporal domain to reduce the background clutter. This paper focuses on the last block, decision making, particularly CFAR-based adaptive thresholding. The final target detection is made by a threshold $(k)$. If the filtered signal intensity of a candidate target $\left(I_{s}\right)$ is larger than $k$, the target is determined to be detected. The original CFAR detector uses the additional information of background statistics such as standard deviation to maintain or reduce the effects of background clutter. Fig. 3 visualizes the operational mechanism of the CFAR detector by changing the threshold adaptively using background statistics, standard deviation $\left(\sigma_{B G}\right)$. Therefore, a new threshold is changed from $k$ to $k^{\prime}=k \cdot \sigma_{B G}$. If the background noise level increases, the threshold automatically increases, which leads to constant false alarms. Fig. 3(a) shows a bright target image in a clean background and corresponding cross-sectional signal intensity plot. In this case, any dim targets can be detected easily because of small standard deviation value of background region. On the other hand, Fig. 3(b) shows another bright target image in a cluttered background and corresponding cross-sectional signal intensity plot. In this case, the target signal should be high enough to be detected because of large standard deviation value of background region.

The original CFAR (O-CFAR) detector has processing flow as shown in Fig. 4(top path). A test image is filtered 


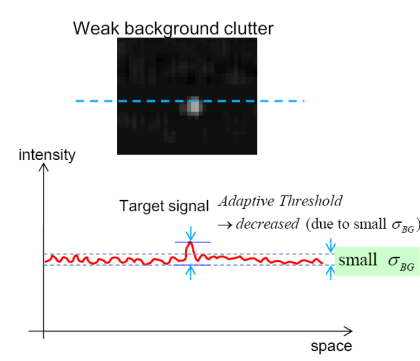

(a)

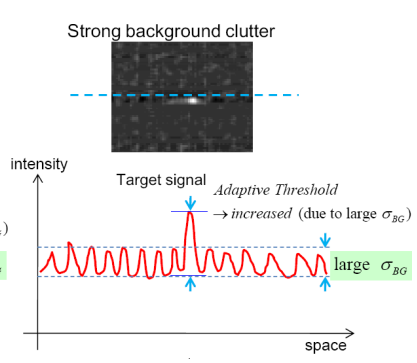

(b)
Fig. 3. Basic concept of a constant false alarm detector to minimize the effect of clutter: (a) weak background clutter case; (b) strong background clutter case.

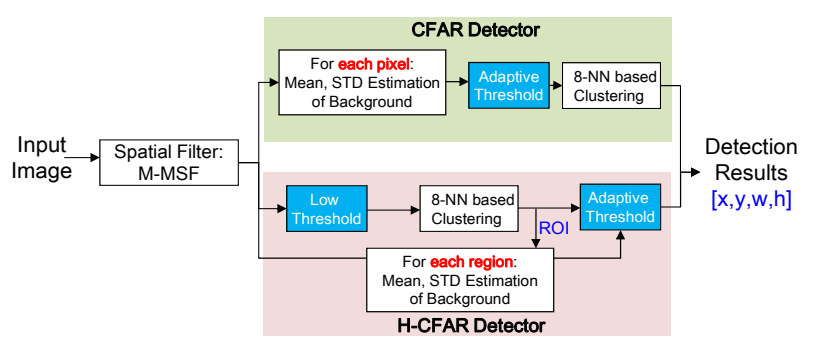

Fig. 4. Comparison of CFAR and H-CFAR detectors in terms of processing flow.

by a specific filter and then the aforementioned adaptive thresholding is applied for each pixel. Final target information ( $\mathrm{x}$ position, $\mathrm{y}$ position, target width, target height) is extracted by clustering. Although the O-CFAR detector shows good detection capability, the processing cost increases enormously due to the pixel-wise statistics estimation. Recently, Kim proposed a computationally efficient method, called hysteresis-threshold-based constant false alarm (H-CFAR) detector [21]. As shown in Fig. 4(bottom path), the H-CFAR uses an adaptive hysteresis threshold consisting of a small threshold for candidate detection and an adaptive CFAR threshold for the final decision. The O-CFAR and H-CFAR show almost the same detection capability except for the processing time. The OCFAR detector searches all the pixels above a thermal noise level. In contrast, the H-CFAR adopts a two kinds of threshding method, called hysteresis threshold. The first small threshold is used for producing region of interest (ROI) and the second adaptive threshold is used for the final decision using background statistics around the ROI region.

Target missing problem of CFAR-based method in closely spaced target detection: O-CFAR or H-CFAR shows good performance on normal detection environments as discussed above. On the other hand, O-CFAR or H-CFAR shows degraded target detection results for closely spaced multitarget scenarios. Fig. 5(b) shows the target detection results using H-CFAR for a APS test image shown in Fig. 5(a). A neighboring decoy hinders the target detection, which is the purpose of the decoy. Likewise, H-CFAR misses
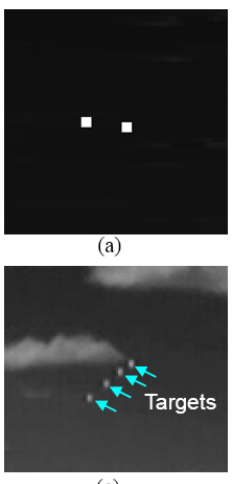

(c)

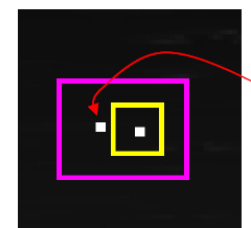

(b)

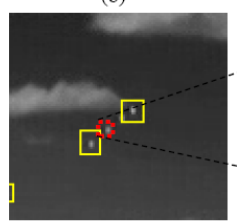

(d)
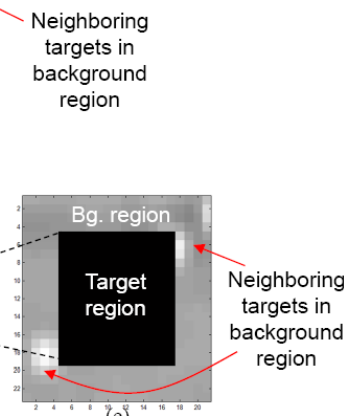

(e)
Fig. 5 Target missing problem of the CFAR-based closely spaced target detection: (a) a cropped APS image with two targets (a decoy, a true target); (b) HCFAR based detection results: a missed target by the neighboring target; (c) a cropped group targets indicated by the arrows; (d) target detection results: two missed targets by neighboring targets, and (e) enlarged probing region of a missed target.

grouped targets in IRST scenario. Fig. 5(d) shows the target detection results using H-CFAR for a APS test image shown in Fig. 5(c). The arrows indicate ground the truth targets and the rectangles represent the targets detected by applying the H-CFAR after a modified mean subtraction filter (M-MSF) [21]. The two center targets were missed by the neighboring targets. Such target missing problem originates from closed spaced targets during an estimation of the background statistics (standard deviation). Fig. 5(e) shows the enlarged second target region (dotted circle in Fig. 5(d)) to analyze such phenomenon. Neighboring targets $\left(1^{\text {st }}\right.$ and $\left.3^{\text {rd }}\right)$ indicated by solid arrows belong to the background region where a CFAR threshold is calculated. The neighboring targets increase the standard deviation of the background, which leads to a target missing problem by the increased threshold value $\left(k^{\prime}=k^{*} \sigma_{B G}\right)$. The proposed context aware-CFAR (CA-CFAR) can solve the problem by intensity sorting-based background pixel selection during the estimation of the background statistics.

The proposed method is quite simple but effective for detecting closely spaced multiple small targets in infrared images.

\section{Proposed Context Aware-CFAR Detection (CA-CFAR)}

The proposed multi-target detection method is based on the H-CFAR detector. Fig. 6 presents the overall flow of context aware-CFAR detector where a context aware block is inserted before the background (BG) statistics estimation to identify neighboring targets in a background region. The spatial filter by M-MSF filter $\left(I_{M-M S F}(x, y)\right)$ provides enhanced target signal by target-background contrast for a 


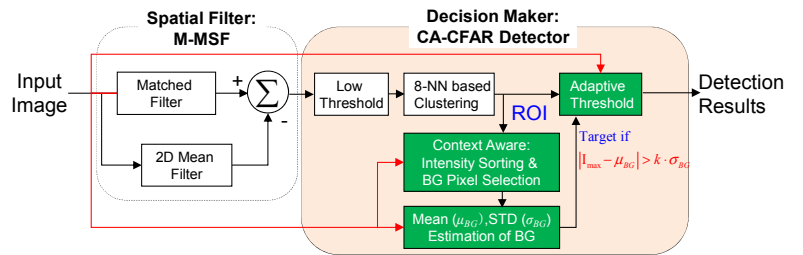

Fig. 6. Proposed context aware-based multiple target detection flow.

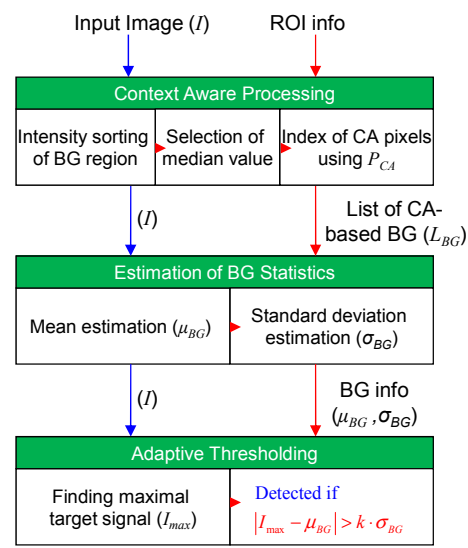

Fig. 7. Details of CA-CFAR detection processing flow.

test image $(I(x, y))$ (see Fig. 2(b)). In the CA-CFAR detector, the candidate target region or region of interest (ROI) can be found by low level thresholding and eightnearest neighbor (8-NN)-based clustering. The background region size is calculated to be three- to four-times the size of the target region. The guard region is just a blank region that is not used in both regions and is set as a two- or threepixel gap (see Fig. 2(c))

The detailed flowchart of CA-based CFAR detection is shown in Fig. 7. It consists of the context aware processing, estimation of background statistics, and adaptive thresholding block. The context aware means that true background pixels can be identified, which helps to find background statistics correctly. The context aware can be performed by an intensity sorting and background pixel selection around a median value. Fig. 8(b) validates this idea for a test image shown in Fig. 8(a). Original background pixels are identified using target ROI information. The intensity sorting histogram (Fig. 8(b)) shows that neighboring target pixels in the background region are usually located at upper position in the intensity ranking. Therefore, a median intensity value can be a starting point of context aware (CA) to exclude neighboring target pixels in a background region. The next step of $\mathrm{CA}$ is to include pixels around the median value using a control parameter, percent of inclusion $\left(P_{C A} \%\right.$, normally $\left.70 \%-80 \%\right)$. In general, infrared images have thermal noise which can be modeled as Gaussian distribution. The target background intensity can be estimated optimally by applying expectation to the Gaussian random variables. The unbiased optimal estimator is the sample average or linear mean. So, the background

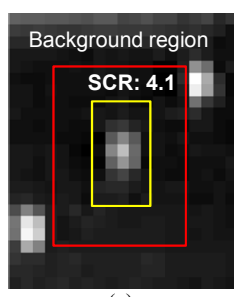

(a)

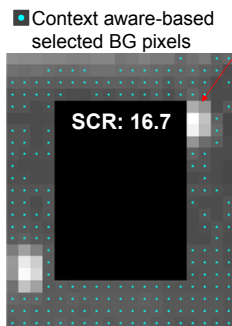

(c)

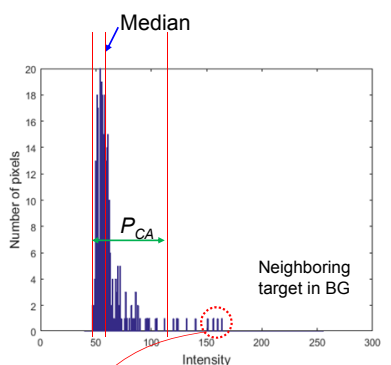

(b)

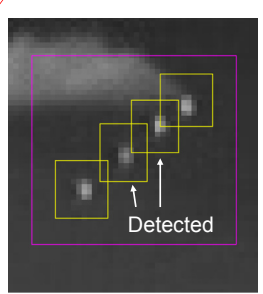

(d)
Fig. 8. Closely spaced multiple target detection procedure: (a) background pixels used in the original H-CFAR (SCR:4.1); (b) context aware (CA)-based analysis of background region using an intensity histogram of the background region; (c) neighboring target rejected background pixels in the proposed CACFAR (SCR: 16.7); (d) final target detection results.

pixel selection of CA can handle both the neighboring target problem and thermal noise reduction. Small dots in Fig. 8(c) represent the selected background pixels (dotts) using the proposed CA method.

Note that the neighboring target pixels were excluded in the background region. The index list $\left(L_{B G}\right)$ of CA-based background pixel is inserted into the following block, estimation of background statistics. The mean $\left(\mu_{B G}\right)$ and standard deviation $\left(\sigma_{B G}\right)$ of the background region can be estimated using Eq. (1) and (2), respectively.

$$
\begin{aligned}
& \mu_{B G}=\underset{(i, j) \in L_{B G}}{\operatorname{mean}}(I(i, j)) \\
& \sigma_{B G}=\underset{(i, j) \in L_{B G}}{S T D}(I(i, j))
\end{aligned}
$$

where STD denotes the abbreviation of standard deviation. $\mu_{B G}$ and $\sigma_{B G}$ are the two key parameter in a CA-CFAR detector because they can control the detection rate and false alarm rate in the closely spaced multiple target scenario. The missed targets can be detected if the parameters $\left(\mu_{B G}, \sigma_{B G}\right)$ is estimated by the CA method.

The final block is to make a decision using an adaptive thresholding method. A probing region is detected as a target if the signal-to-background $\left(\left|I_{\max }-\mu_{B G}\right|\right)$ is greater than the second threshold $k \cdot \sigma_{B G}$, as defined in Eq. (3).

$$
\begin{aligned}
& \text { Detected if } \\
& \left|I_{\max }-\mu_{B G}\right|>k \cdot \sigma_{B G}
\end{aligned}
$$

where $I_{\max }$ denotes maximum target signal obtained using 
Eq. (4), where $T$ represents an index set of the target region.

$$
I_{\max }=\max _{(i, j) \in T}(I(i, j))
$$

Note that Eq. (3) can be converted to signal-to-clutter ratio (SCR) if the equation is divided by $\sigma_{B G}$, as defined in Eq. (5).

$$
\mathrm{SCR}=\frac{\left|I_{\max }-\mu_{B G}\right|}{\sigma_{B G}}>k
$$

Fig. 8(c) presents the effect of the proposed CA-CFAR in closely spaced multiple target detection. It can reject the neighboring target pixels using $P_{C A}=70 \%$ and showed a enhanced SCR of 16.7 for the same target. Note that correct background statistics were obtained using the proposed CA-based background statistics estimation. Fig. 8(d) shows CA-CFAR based detection results where the two missed targets in Fig. 5(d) were detected correctly. Therefore, the proposed new CA-CFAR can increase the detection rate by removing the effects of the neighboring targets during the calculation of the background statistics. Because the final threshold ( $k$ ) in Eq. (3) or Eq. (5) controls the detection rate and false alarm rate, it can be changed depending on the operation scenarios.

\section{Experimental Results}

The proposed CA-CFAR was compared with the baseline method, H-CFAR [21]. The input images were filtered using the same spatial filter, M-MSF. The two test image sets were prepared to validate the performance of the proposed method. One is the real infrared image sequence of Seoul air show, consisting of four F-15K fighters with adjacent formation flight in strong cloud clutter and acquired using a Cedip, LWIR camera (Set 1). The other was generated by commercial software called OKTAL-SE (Set 2) [22]. OKTAL-SE is the only simulator that can synthesize both passive (IR) and active (Synthetic Aperture Radar). The scenario program can select the background and target trajectory and the SE-RAY-IR then
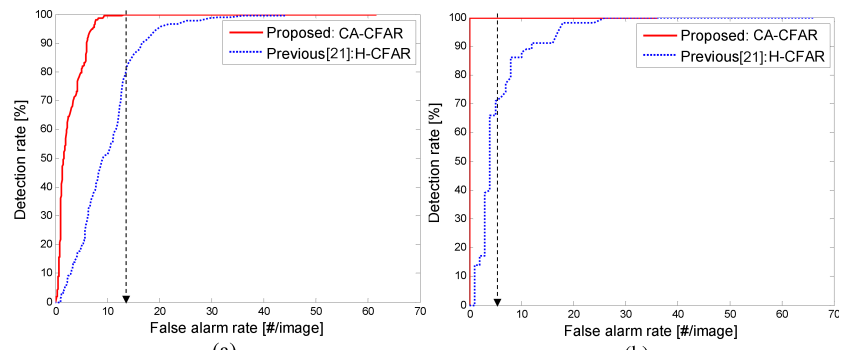

(a)

(b)

Fig. 9. Comparative detection performance for the proposed CA-CFAR and H-CFAR given the test Set 1 (a), Set 2 (b). synthesizes the IR sequences using the ray tracing method. For an active protection system (APS) in military applications, two targets (one is the real target, the other is a decoy) were inserted and the incoming target distance was $1.23 \mathrm{~km}$ at Mach 6 .

The detection performance was compared using the receiver operating characteristic (ROC) curve using the detection rate (DR) and false alarm rate (FAR) by varying the adaptive threshold $(k)$. The low level threshold was set to 10. As shown in Fig. 9, the proposed method outperforms the other in terms of ROC curve area for test Sets 1 and 2. Table 1 lists the statistical performance comparisons of the proposed CA-CFAR and previous $\mathrm{H}$ -

Table 1. Statistical performance comparisons of the closely space multiple target detection methods (DR: detection rate, FAR: number (\#) of false alarms per image).

\begin{tabular}{c|c|c|c|c}
\hline Test set & \multicolumn{2}{|c|}{ Set 1 (53 images) } & \multicolumn{2}{c}{ Set 2 (50 images) } \\
\hline Method & $\begin{array}{c}\text { CA-CFAR } \\
\text { (proposed) }\end{array}$ & $\begin{array}{c}\text { H-CFAR } \\
{[21]}\end{array}$ & $\begin{array}{c}\text { CA-CFAR } \\
\text { (proposed) }\end{array}$ & $\begin{array}{c}\text { H-CFAR } \\
{[21]}\end{array}$ \\
\hline Threshold $(k)$ & 8.1 & 5.2 & 13.9 & 7.5 \\
\hline DR [\%] & $\mathbf{1 0 0 . 0}$ & 77.7 & $\mathbf{1 0 0 . 0}$ & 71.0 \\
\hline FAR [\#/image] & $\mathbf{1 3 . 2}$ & 13.2 & $\mathbf{5 . 0}$ & 5.0 \\
\hline
\end{tabular}
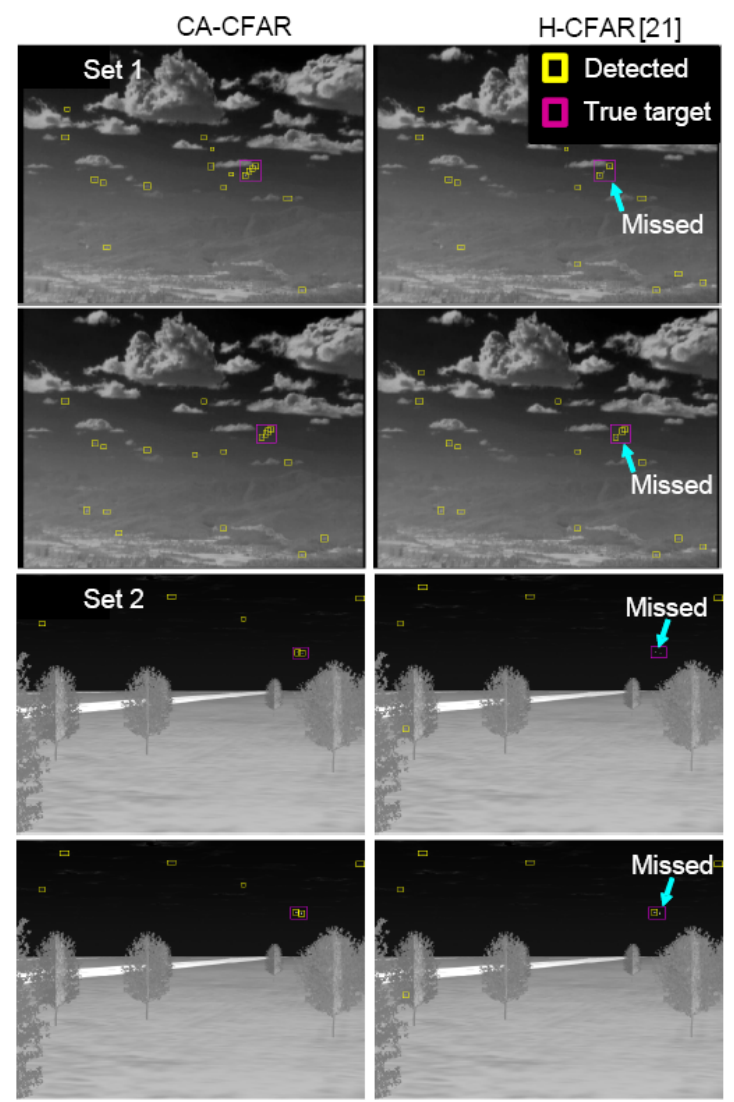

Fig. 10. Comparative analysis of the closely spaced multiple target detection for the test Set 1 and Set 2. Small yellow rectangles represent detected results and large magenta rectangles represent ground truths. 

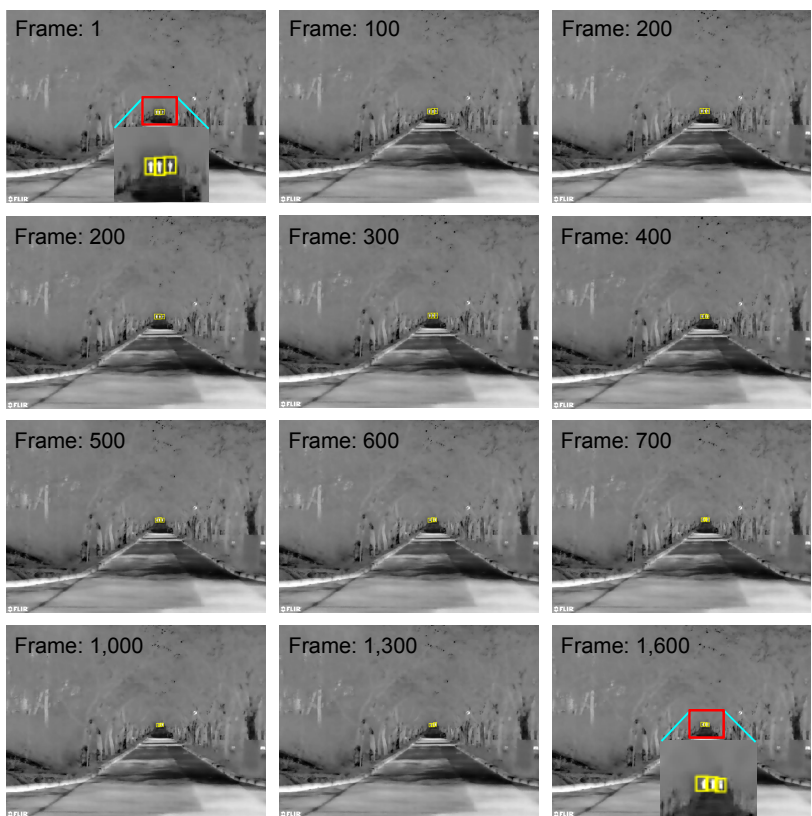

Fig. 11. CA-CFAR application results to the remote people detection scenario.

CFAR [21] given the same false alarm rate (FAR) indicated by the arrows in Fig. 9. According to the results, the proposed CA-CFAR produced a much larger number of correct detections than the baseline method, H-CFAR [21]. Fig. 10 presents the closely space multiple target detection results of cluttered images, where the small rectangles represent the detection and large rectangles ground truth locations. As indicated by the arrows, the H-CFAR often misses the closely spaced multiple targets due to the high background standard deviation. Note the superior detection performance of the CA-CFAR based method in the adjacent multi-target detection scenarios.

An additional experiment was conducted to validate the general capability of the proposed CA-CFAR detector for closely spaced small infrared targets. The test scenario is as follows. Three people run on the road at $300 \mathrm{~m}$ distance and a FLIR T620 (field of view: $25^{\circ}$, long wave infrared, $640 \times 480$ resolution) mounted on a car records the scene at night time. The total number of test frames is 1,600 and a low threshold was set as 100 and an adaptive threshold parameter $k$ was set 5 to detect the people. Fig. 11 shows the partial detection results for the closely moving people. The proposed CA-CFAR detector can localize multiple people correctly for the whole sequence.

\section{Conclusion}

The adaptive threshold-based small target detection method such as CFAR normally uses background statistics (standard deviation) to produce constant false alarms regardless background noise level. Although the con- ventional method works well in normal scenarios, it fails to detect closely spaced multiple targets. This paper proposed a new context aware-based multiple target detection method, which is simple but powerful detection capability for small infrared targets using a intensity sorting-based background pixel selection in a background statistics estimation. As validated by a set of quantitative experiments, it can effectively find the true targets with the formation flight. Additional experiment on the remote people detection showed good detection performance. Because the proposed method can increase the computational cost by $15 \%$, it can be used for real-time applications of stationary and moving infrared camera platforms because of the simplicity of the algorithm with powerful detection capability for dense target detection.

\section{Acknowledgements}

This research was supported by Basic Science Research Program through the National Research Foundation of Korea (NRF) funded by the Ministry of Science, ICT \& Future Planning (NRF-2014R1A2A2A01002299).

\section{References}

[1] T. J. Meyer, “Active protective systems: impregnable armor or simply enhanced survivability?" Armor, May-June: 7-11, 1998.

[2] A. N. de Jong, "IRST and perspective," In Proc. of SPIE, vol. 2552, pages 206-213, 1995.

[3] S. Kim, "Double layered-background removal filter for detecting small infrared targets in heterogeneous backgrounds," J. Infrared Milli. Terahz Waves, 32(1): 79-101, 2011.

[4] H. Sang, X. Shen, and C. Chen, "Architecture of a configurable 2-D adaptive filter used for small object detection and digital image processing," Optical Engineering, 48(8): 2182-2189, 2003.

[5] Y. L. Wang, J. M. Dai, X. G. Sun, and Q. Wang, “An efficient method of small targets detection in low SNR," J. of Physics: Conference Series, 48: 427-430, 2006.

[6] Y. S. Jung and T. L. Song, "Aerial-target detection using the recursive temporal profile and spatiotemporal gradient pattern in infrared image sequences," Optical Engineering, 51(6):066401, 2012.

[7] S. Kim, "High-speed incoming infrared target detection by fusion of spatial and temporal detectors," Sensors, 15(4):7267-7293, 2015.

[8] B. Zhang T. Zhang, Z. Cao, and K. Zhang, "Fast new small-target detection algorithm based on a modified partial differential equation in infrared clutter," Opt. Eng., 46(10), 2007. 
[9] R. C. Warren, Detection of distant airborne targets in cluttered backgrounds in infrared image sequences, 2002. Ph.D. Thesis, University of South Australia.

[10] M. S. Longmire, E. H. Takken, "LMS and matched digital filters for optical clutter suppression," Applied Optics, vol. 27, 1141-1159, 2003.

[11] T. Soni, J. R. Zeidler, W. H. Ku, "Performance Evaluation of 2-D Adaptive Prediction Filters for Detection of Small Objects in Image Data," IEEE Trans. Image Processing, vol. 2, 327-340, 1993.

[12] J. F. Rivest, R. Fortin, "Detection of Dim Targets in Digital Infrared Imagery by Morphological Image Processing," Opt. Eng., vol. 35, 1886-1893, 1996.

[13] A. Kojima, N. Sakurai, J. I. Kishigami, "Motion detection using 3D-FFT spectrum," IEEE International Conference on Acoustics, Speech, and Signal Processing (ICASSP), vol. 5, pages 213-215, 1993.

[14] R. N. Strickland, H. I. Hahn, "Wavelet Transform Methods for Object Detection and Recovery," IEEE Trans. Image Processing, vol. 6, pages 724-735, 1997.

[15] G. Boccignone, A. Chianese, A. Picariello, "Small target detection using Wavelets," International Conf. on Pattern Recognition, pages 1776-1778, 1998.

[16] Z. Ye, J. Wang, R. Yu, Y. Jiang, Y. Zou, "Infrared clutter rejection in detection of point targets," Proc. of SPIE, vol. 4077, pp. 533-537, 2002.

[17] Z. Zuo, T. Zhang, "Detection of Sea Surface Small Targets in Infrared Images based on Multi-level Filters," Proc. of SPIE, vol. 3544, pp. 372-377, 1999.

[18] L. Yang, J. Yang, K. Yang, “Adaptive Detection for Infrared Small Target under Sea-sky Complex Background," Elec. Lett., vol. 40, pages 1083-1085, 2004.

[19] M. Fernandez, "Adaptive single-frame superresolution for detecting closely spaced IR targets in clutter," IEEE Transactions on Aerospace Electronic Systems, 50(4): 2489-2499, 2014.

[20] F.-Y. Xu, G.-H. Gu, andW. Qian, "The research and implementation of CFAR in infrared small target detection," Proc. of SPIE, 8193:81931N, 2011.

[21] S. Kim and J. Lee, "Small infrared target detection by region-adaptive clutter rejection for sea-based infrared search and track," Sensors, 14(7):13210-13242, 2014.

[22] J. Latger, T. Cathala, and N. Douchin A. L. Goff, "Simulation of active and passive infrared images using the SE-WORKBENCH," Proc. of SPIE, 6543:654302, 2007.

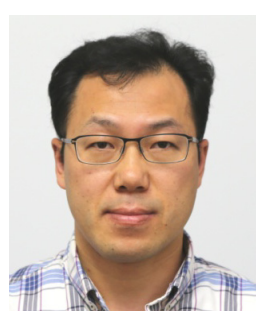

Sungho Kim $\mathrm{He}$ received the BS degree in electrical engineering from Korea University, Korea in 2000 and the MS, PhD degrees in electrical engineering and computer science from Korea Advanced Institute of Science and Technology, Korea in 2002, 2007, respectively. During 2007 and 2010, he was a senior researcher in the Electro-Optics Laboratory at the agency for defense development (ADD). Since 2010, he has been a professor of Electronic Engineering at Yeungnam University. His current research interests include small infrared target detection and target recognition.

Jin-Ju Won She received the BS degree in electronic engineering from Yeungnam University, Korea in 2015. Currently, she is pursuing her MS degree focusing on the ground target detection, recognition and SAR/IR fusionbased target recognition. 